\title{
PROPOSAL OF A LOW COST HIGH PERFORMANCE EDUCATIONAL MOBILE ROBOT: AN RPI AND ARDUINO APPROACH
}

\author{
JOSÉ GONÇALVES * \\ Research Centre in Digitalization and Intelligent Robotics (CeDRI), Instituto \\ Politécnico de Bragança, Campus de Santa Apolónia, 5300-253 Bragança, Portugal \& \\ INESC-TEC \\ *E-mail: goncalves@ipb.pt
}

\author{
ANDRÉ F. PINTO, VITOR H. PINTO, PAULO COSTA \\ Faculty of Engineering of the University of Porto \& INESC-TEC \\ E-mail: \{ee12007,vitorpinto,paco\}@fe.up.pt
}

\begin{abstract}
In this paper the proposal of a low cost high performance educational mobile robot is described. The robot is based on an Arduino, applied in the low level control, while the high level control loop is carried out by an RPI running an object pascal application. The described robot was prototyped in order to have a competitive participation in the Robotic Day Line Follower 2017 competition, taking advantage of the RPI capabilities. The RPI allows the use of higher performance sensors, when compared with the most common standard approaches based on a single 8 bit RISC micro-controller, having as disadvantage the inevitable robot size increase, which compromises in certain situations the robot maneuverability and increases the power consumption. The robot is equipped with DC Motors, the chosen line follower sensor is the picamera and for the obstacle detection sonar sensors are used.
\end{abstract}

Keywords: Robotics, Education, Prototyping

\section{Introduction}

Robotic Day is a very successful international robot competition, that is held annually in Prague. This robot competition has been active during the last 14 years and still continues to grown in number of participants, teams, visitants and competitions ${ }^{1}$. In this paper it is described the proposal of a low cost high performance educational mobile robot, that was prototyped in order to have a competitive participation in the Robotic Day Line Follower 2017 competition. The Line Follower Robotic Competition can be

H. Montes et al. (eds.), CLAWAR 2018: 21st International Conference on Climbing and Walking Robots and the Support Technologies for Mobile Machines,

Robotics Transforming the Future

(C) ELSEVIER 
seen in Figure 1 and the proposed robot in Figure 2. The robotic competitions present standard problems that can be used as a benchmark, in order to evaluate and to compare the performances of different approaches. Although there are many robotic competitions, there is the need to create new ones, in order to solve new challenges ${ }^{23456}$. The robot prototyping and programming, in a competition context, can play an important role in education due to its inherent multidisciplinary approach, which can motivate students to bridge different technological areas. It can also play an important role in research and development, because it is expected that its outcomes will later be transferred to real-world problems in manufacturing or service robots.

The proposed robot is based on an Arduino, applied in the low level control, while the high level control loop is carried out by an RPI running an object pascal application. The RPI allows the use of higher performance sensors, when compared with the most common standard approaches based on a single 8 bit RISC micro-controller, having as disadvantage the inevitable robot size increase, which compromises in certain situations the robot maneuverability and increases the power consumption.

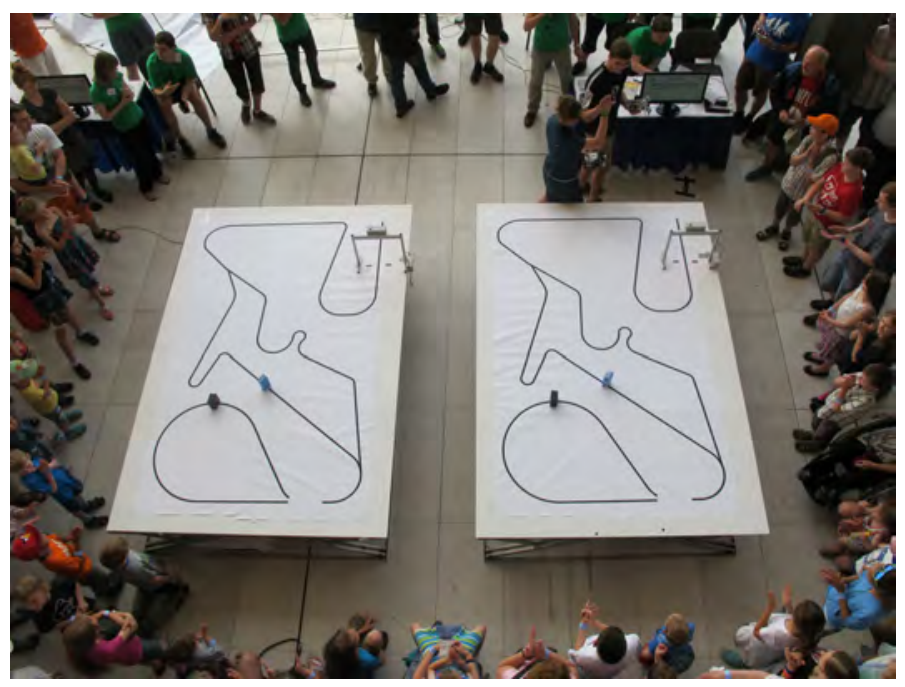

Fig. 1: Line Follower Robotic Competition

The robot has to run over the course and follow black line. During the qualifications robots proceed to next round if they successfully pass all obstacles and finish the run. Robots with the best time in the last 
qualification round qualify for the finales. In the finale part, the races will be held on a knock-out basis. In case of a tie, a repeated race may be ordered by the organizers. There may be some obstacles on the track:

- An object: There could be an object laid on the track. The robot may touch it but it must not move it.

- Line cut-off: The line may be discontinued at any place for max. $20 \mathrm{~cm}$.

- Line split and join: Situation like line split and join could exist.

The described robot was qualified for the finals of the Robotic Day 2017 edition, it were qualified 8 robots, from a total of 53 participants, the robot performance can be observed in the video available in ${ }^{7}$.

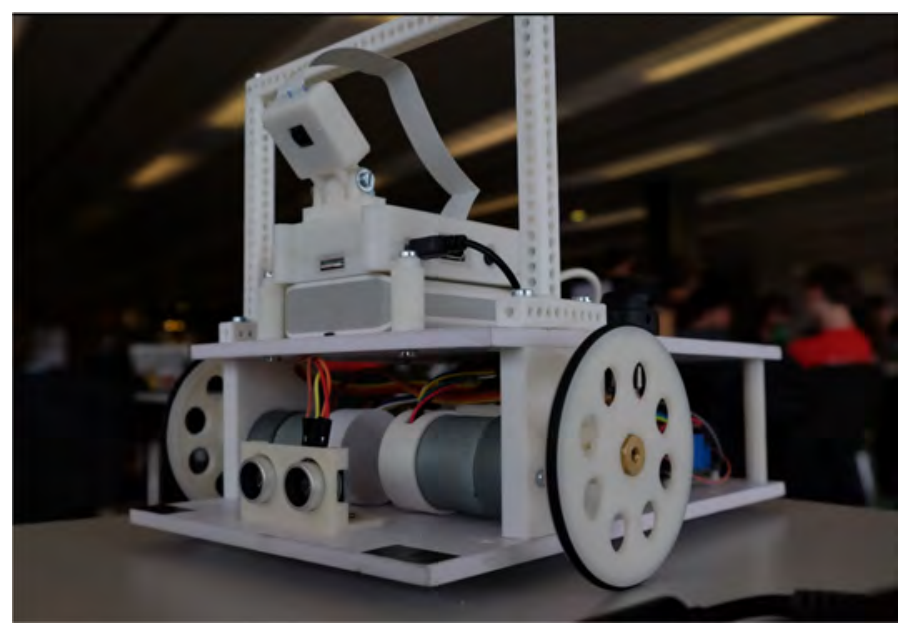

Fig. 2: Robot Prototype

\section{Robot Prototype Description}

The prototyped mobile robot consists in a 3D printed small prototype, that uses inexpensive hardware, such as DC motors, an Arduino, an RPI3, sonars and a picamera to sense the line, the robot prototype components are shown in Figure 3. The 3D printer models, that were developed in order to prototype the robot, are presented in Figure 4, where it can be seen the $3 \mathrm{D}$ models for the robot chassis, wheels, RPI case and picamera support and hinge $3 \mathrm{D}$ models. 


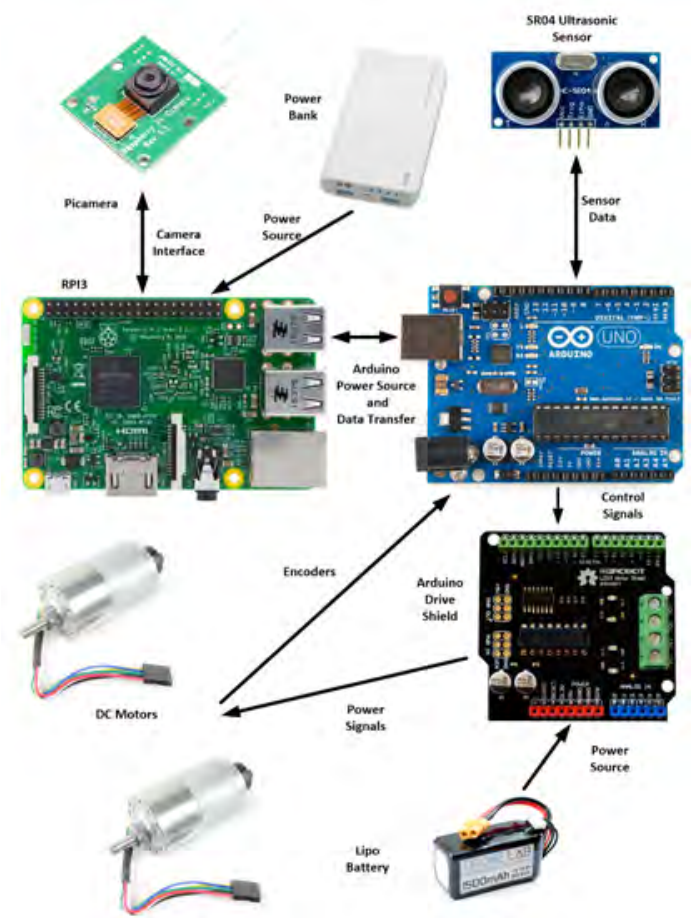

Fig. 3: Robot Prototype Components
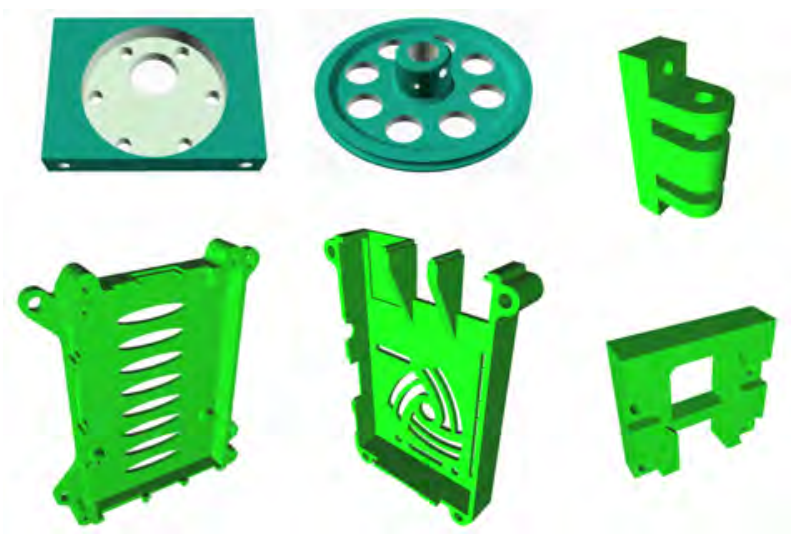

Fig. 4: Robot Prototype 3D Printer Models.

\subsection{Sensors}

In this section are presented the sensors that are applied in the robot. Initially is presented the computer vision sensor, which is a common sensor 
in mobile robotics, and it is also presented the sonar being used for obstacle $\operatorname{detection}^{8}$.

\subsubsection{Computer Vision}

A Raspberry Pi camera was used to detect the line that must be followed. While the standard approach is to use an array of IR sensors, it is very inflexible and its resolution is limited. By implementing a virtual line sensor from the image pixels, an higher resolution can be achieved and multiple virtual sensors can easily be implemented.

The current setup has a resolution of 320 x 240 with a frame rate of 30 frames per second. The acquired image covers an area in front of the robot with an width of $25 \mathrm{~cm}$ and a length of $35 \mathrm{~cm}$. The virtual line sensors can be placed anywhere inside that area. A dedicate application handles the image acquisition, that can be seen in Figure 5, the virtual line sampling and processing. For each virtual sensor the line position is calculated and its width is used to access the measure confidence. There is some degree of adaptability on the luminance thresholds to deal with different light conditions.

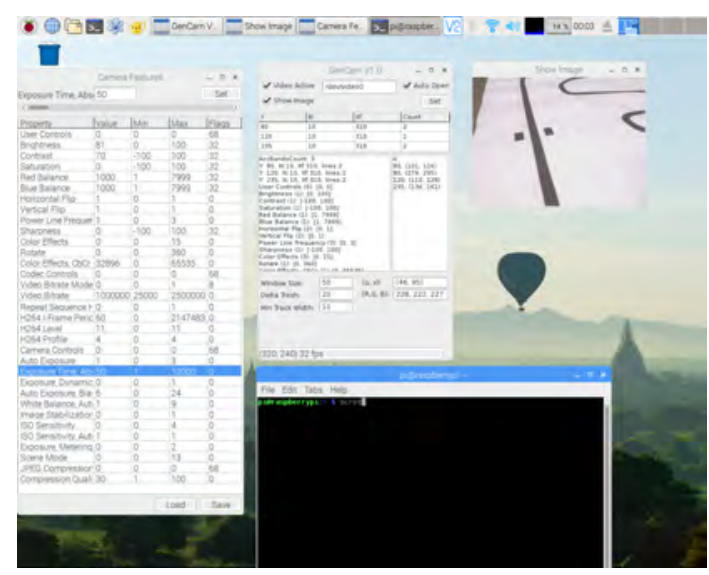

Fig. 5: Virtual Line Sensor Software

\subsubsection{Sonar}

The chosen sensor used to detect the obstacles is the Ultrasonic ranging module HC - SR04, shown in Figure 3, which provides $2 \mathrm{~cm}-400 \mathrm{~cm}$ 
measurement function, with a ranging accuracy that can reach to $3 \mathrm{~mm}$. The modules include ultrasonic transmitters, receiver and control circuit.

\subsection{Actuators}

The applied DC motors have built in encoders which are very important in mobile robots in order to obtain the closed loop Velocity Control and also to estimate the robot odometry calculation and the applied drive is the DFRobot L293 Arduino Shield.

\section{Controller description}

Following a line with a robot based on the differential kinematics is a classical introductory experiment that allows students to be introduced to the challenges of mobile robotics ${ }^{1011}$. Understanding the concepts of sensor, actuator and locomotion are the primary goals of this challenge based on the control of a reactive robot $^{9}$. In this section it will be described the developed prototype controller.

\subsubsection{Line Following}

The goal of the robot is to follow a line as fast as possible, for that purpose it is necessary to maintain the highest possible linear velocity, with an angular velocity that is proportional to the path curvature. With the picamera, applied as line sensor, it is possible to know at each sample time the robot posture in relation to the line. A detailed description of the applied line following controller algorithm can be found in ${ }^{12}$.

\subsubsection{Obstacle avoidance}

In order to the robot avoid and contour an obstacle the state machine observed in Figure 6 was used. Initially the robot, when an obstacle is detected, rotates with a null linear velocity and then, using odometry, the robot performs a smooth trajectory without using the camera data to navigate, finally the camera data is used and if a line is detected the robot will navigate using the standard line following controller, as described in in ${ }^{12}$.

\subsubsection{Line Interruption}

Each virtual sensor can detect the line presence or absence, by analyzing the line total dynamic range. The line absence can be detected by testing if 


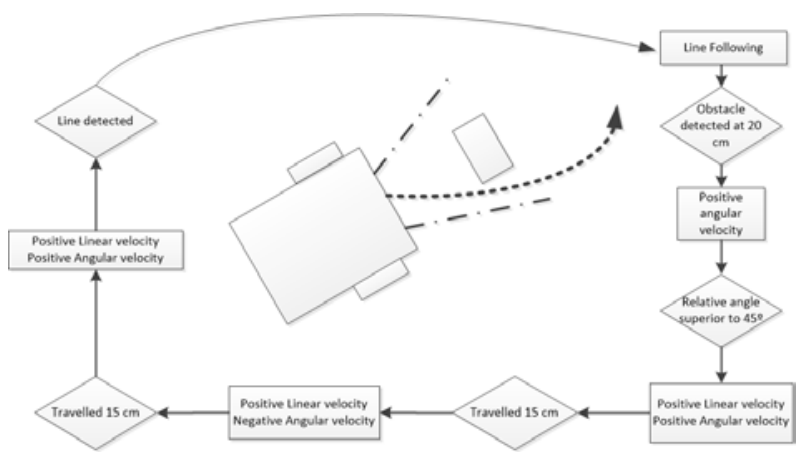

Fig. 6: Obstacle avoidance State Machine

the minimum sampled value is above a certain threshold, that means there isn't a black zone on the line. If the condition is met for a few samples the line interruption event is triggered and the current direction is frozen, that allows the robot to travel until it meets the line again.

\section{Conclusions and Future Work}

In this paper it is described the proposal of a low cost high performance educational mobile robot. The robot is based on an Arduino, applied in the low level control, while the high level control loop is carried out by an RPI running an object pascal application.

The described robot was prototyped in order to have a competitive participation in the Robotic Day Line Follower 2017 competition. The RPI apply allows the use of higher performance sensors, when compared with the applied in the most common standard approaches based on a single 8 bit RISC micro-controller, having as disadvantage the inevitable robot size increase, which compromises in certain situations the robot maneuverability and increases the power consumption.

The robot is equipped with DC Motors with built in encoders, which allows the apply of velocity closed loop control and the necessary data to the odometry calculation. The ability to calculate the robot odometry was very important, because the robot had, in some situations, to perform trajectories without sensing the line. The chosen line follower sensor is the picamera, providing a very flexible sensor based on image processing.

The authors intend to evolve the software robustness of the proposed robot in order to participate in the 2018 Robotic Day Line Follower Competition edition. 


\section{Aknowledgements}

This work is financed by the ERDF European Regional Development Fund through the Operational Programme for Competitiveness and Internationalisation - COMPETE 2020 Programme within project POCI-01-0145FEDER-006961 and by National Funds through the FCT Fundação para a Ciência e a Tecnologia (Portuguese Foundation for Science and Technology) as part of project UID/EEA/50014/2013.

\section{References}

1. Richard Balogh, Grzegorz Granosik, Valery Kasyanik, David Obdrzalek. Experiences from the Series of International Robotics Workshops. Computer Science and Information Technology, 4 , 126 - 133. doi: 10.13189/csit.2016.040304, 2016.

2. A. Eguchi RoboCupJunior for promoting STEM education, 21st century skills, and technological advancement through robotics competition, Robotics and Autonomous Systems, 2016.

3. E. Pagello and E. Menegatti and A. Bredenfeld and P. Costa and T. Christaller and A. Jacoff and D. Polani and M. Riedmiller and A. Saffiotti and E. Sklar and T. Tomoichi, Robotic Competition Based Education in Engineering,AI Magazin, 2004

4. F. Ribeiro and I. Moutinho and P. Silva and C. Fraga and N. Pereira, Controlling Omni-Directional Wheels of a Robocup MSL Autonomous Mobile robot, Scientific Meeting of the Portuguese Robotics Open, 2004

5. B. Browning and J. Bruce and M. Bowling and M. Veloso, USTP: Skills, tactics and plays for multi-robot control in adversarial environments, IEEE Journal of Control and Systems Engineering, 2005

6. R. Nakanishi and J. Bruce and K. Murakami and T. Naruse, and M. Veloso, Cooperative 3-robot passing and shooting in the RoboCup Small Size League, Proceedings of the RoboCup Symposium, 2006

7. Gonçalves, J., Robotic Day 2017, Robot: LF@RD17, TEAM: PT@RDLF17, ORGANIZATION: IPB/FEUP, https://www.youtube.com/watch?v=6qnDiBPg2Ms

8. Borenstein, J., Everett, H., Feng, J. 'Where am I?' Sensors and Methods for Mobile Robot Positioning Technical Report, The University of Michigan 1996

9. Pakdaman, M. Design and Implementation of Line Follower Robot Second International Conference on Computer and Electrical Engineering 2009

10. Gregory Dudek and Michael Jenkin Computational Principles of Mobile Robotics Cambridge University Press 2000

11. H. Choset, K. Lynch, S. Hutchinson, G. Kantor, W. Burgard, L. Kavraki, S. Thrun, Principles of Robot Motion: Theory, Algorithms, and Implementations, MIT Press, 2005.

12. Gonçalves, J and Costa, P Differential mobile robot controller study: A low cost experiment based on a small arduino based prototype, 25th Mediterranean Conference on Control and Automation, 2017 